\title{
Spontaneous radiation emission from short, high field strength magnetic devices
}

\author{
G. A. Krafft \\ Center for Advanced Studies of Accelerators, Jefferson Lab, Newport News, Virginia 23608, USA
}

(Received 1 September 2005; published 5 January 2006)

\begin{abstract}
Since the earliest papers on undulators were published, it has been known how to calculate the spontaneous emission spectrum from short undulators when the magnetic field strength parameter is small compared to unity, or in "single" frequency sinusoidal undulators where the magnetic field strength parameter is comparable to or larger than unity, but where the magnetic field amplitude is constant throughout the undulator. Fewer general results have been obtained in the case where the magnetic device is both short, i.e., the magnetic field strength parameter changes appreciably throughout the device, and the magnetic field strength is high enough that ponderomotive effects, radiation retardation, and harmonic generation are important physical phenomena. In this paper a general method is presented for calculating the radiation spectrum for short, high-field magnetic devices. It is used to calculate the emission from some designs of recent interest.
\end{abstract}

DOI: 10.1103/PhysRevSTAB.9.010701

PACS numbers: 41.60.Ap, 41.60.Cr, 41.75.Lx

\section{INTRODUCTION}

This paper deals with the problem of calculating the spontaneous electromagnetic radiation spectrum generated by electrons passing through magnetic devices. Using previously known results [1], the radiation spectrum can be calculated exactly, in the far-field limit, as long as the magnetic field is small enough that the magnetic field strength parameter is much less than unity throughout the device. In brief, the spectrum is obtained by Fourier transforming the transverse velocity induced by the magnetic field in the electron, and suitably up-shifting the radiation frequency distribution by the relativistic Doppler effect. Such a prescription was discovered during attempts to use synchrotron light from protons or other heavy particles at high energy as a beam diagnostic [2], where it was uniformly true that the magnetic field patterns generating the radiation had to be considered "short," i.e., the field strength parameter could change substantially during the transit through the region producing the synchrotron light.

The problem of harmonic generation [3,4] in intense laser Thomson scattering has been recently reinvestigated [5]. As the laser pulses being scattered are not uniform in the longitudinal direction in realistic experiments, a semianalytic theory of the spontaneous emission from intense pulsed laser beams was developed, where a key aspect of the calculation was to allow the intensity, or equivalently the effective field strength parameter, to vary throughout the laser pulse [6]. A phenomenon called ponderomotive broadening, where the spectrum of the emitted radiation is much wider than the spectrum observed at low-field strength parameter because of the slowing down of electrons by the ponderomotive effect of the pulsed laser, was observed. The broadening is especially pronounced at higher harmonic numbers, eventually causing the radiation spectrum to become continuous. Because the physics of the emission of radiation by magnetic devices is practically identical to that of Thomson scattering, one might expect a formalism similar to that used in the intense pulsed laser Thomson scattering problem to apply to the problem of short magnets operated at high-field strengths. In this paper some results of conventional insertion device theory are generalized in this manner, and used to compute the spontaneous radiation spectrum in some cases of recent interest. It is shown that spectral broadening will be exhibited in some designs.

In the first part of the paper the electron dynamics model used in the calculations is introduced. In the second part of the paper the dynamical equations are used to determine the emission spectrum. In the third part of the paper a generalization of the usual relativistic Larmor emission formula is given. In the final part of the paper, the preceding work is used to analyze a " $\frac{1}{2}$ " period intense magnetic device and a Gaussian amplitude modulated magnetic device. In the latter case it is shown that ponderomotive broadening can significantly affect the emission from the device.

\section{ELECTRON DYNAMICS IN SHORT MAGNETS}

Following standard practice in elementary insertion device theory we shall assume that the electron dynamics is dominantly defined by a one-dimensional static transverse magnetic field. By this statement is meant that the vector potential describing the magnetic field depends on the single longitudinal coordinate $z$, where the $z$ axis of the coordinates is aligned along the initial electron motion. Therefore for linearly polarized magnetic devices the vector potential consists of a single component in the direction transverse to the magnetic field direction, whereas for circularly polarized devices the vector potential has two 
components phase shifted by a quarter oscillation with respect to each other in the longitudinal coordinate. By taking such a model, the effects of linear focusing by the device are neglected because the magnetic field is uniform in the transverse direction, and it is implicitly assumed that the electron beam is smaller than any scale length changes in the magnetic field.

The relativistic equations of motion of an electron in such a magnetic field reduce to

$$
\begin{aligned}
& \gamma(z)=\gamma_{0}, \quad \gamma m v_{x}(z)-e A_{x}(z) / c=C_{x} \\
& \gamma m v_{y}(z)-e A_{y}(z) / c=C_{y}
\end{aligned}
$$

where $\gamma$ is the usual relativistic factor; $m$ is the invariant (rest) mass of the electron; $v_{x}$ and $v_{y}$ denote the transverse components of the lab frame velocity; $-e$ is the charge of the electron; $A_{x}$ and $A_{y}$ denote the transverse components of the vector potential; $c$ denotes the speed of light; and $\gamma_{0}$, $C_{x}$, and $C_{y}$ are constants of the motion. The first equation is simply energy conservation and the other two equations are simply a statement of conservation of canonical momentum. Because the device does not stretch to infinity, the vector potential components may be assumed to vanish as $z \rightarrow \pm \infty$. If there is no transverse motion of the electron before entering the magnet, the canonical momenta $C_{x}$ and $C_{y}$ are trivially evaluated to be zero, and

$$
\begin{aligned}
& \gamma_{0} \beta_{x}(z)=\frac{e A_{x}(z)}{m c^{2}} \equiv a_{x}(z), \\
& \gamma_{0} \beta_{y}(z)=\frac{e A_{y}(z)}{m c^{2}} \equiv a_{y}(z),
\end{aligned}
$$

where $\vec{\beta}$ is the velocity normalized to the speed of light and $a_{x}$ and $a_{y}$ give the vector potential in unitless quantities. Using energy conservation and these two equations, the normalized longitudinal velocity is found to be

$$
\begin{aligned}
\beta_{z}(z) & =\sqrt{1-\frac{1}{\gamma_{0}^{2}}\left[1+a_{x}^{2}(z)+a_{y}^{2}(z)\right]} \\
& =\sqrt{\beta_{z 0}^{2}-\frac{a_{x}^{2}(z)+a_{y}^{2}(z)}{\gamma_{0}^{2}}}
\end{aligned}
$$

where $\beta_{z 0}$ is the longitudinal velocity before entering the magnetic device. Because Eq. (3) is an exact expression for the longitudinal velocity as a function of $z$, and because the normalized transverse velocities are given explicitly as functions of $z$, the transverse orbit may be easily obtained as a function of the independent variable $z$, instead of the more usual $t$ :

$$
\begin{aligned}
\frac{d(c t)}{d z} & =\frac{1}{\beta_{z}} \rightarrow c t(z)=\lim _{L \rightarrow-\infty} \frac{L}{\beta_{z 0}}+\int_{L}^{z} \frac{d z^{\prime}}{\beta_{z}\left(z^{\prime}\right)} \\
& =\frac{z}{\beta_{z 0}}+\int_{-\infty}^{z}\left[\frac{1}{\beta_{z}\left(z^{\prime}\right)}-\frac{1}{\beta_{z 0}}\right] d z^{\prime}, \\
\frac{d x}{d z} & =\frac{\beta_{x}}{\beta_{z}} \rightarrow x(z)=\int_{-\infty}^{z} \frac{a_{x}\left(z^{\prime}\right)}{\beta_{z}\left(z^{\prime}\right) \gamma_{0}} d z^{\prime}, \\
\frac{d y}{d z} & =\frac{\beta_{y}}{\beta_{z}} \rightarrow y(z)=\int_{-\infty}^{z} \frac{a_{y}\left(z^{\prime}\right)}{\beta_{z}\left(z^{\prime}\right) \gamma_{0}} d z^{\prime} .
\end{aligned}
$$

It has been assumed, without loss of generality, that the transverse offsets vanish as $z \rightarrow-\infty$ and that the electron orbit would pass $z=0$ at $t=0$ had there been no reduction in the longitudinal velocity from the magnetic field. Given the spatial dependence of the vector potential describing the magnetic field, the complete orbit through the magnetic device has been obtained. The integrals found are easily evaluated numerically.

\section{SPONTANEOUS ELECTROMAGNETIC EMISSION SPECTRUM}

We begin by noting that in the case of linear polarization, as in Thomson scattering calculations, it is particularly convenient to resolve the polarization of the emitted radiation into the polarization parallel and perpendicular to the plane of emission. Beginning with the usual result for the spectral angular flux into a given solid angle in the far field [7]

$$
\begin{aligned}
\frac{d^{2} E}{d \omega d \Omega}= & \frac{e^{2}}{8 \pi^{2} c} \\
& \times\left|\int_{-\infty}^{\infty} \frac{d}{d t}\left[\frac{\vec{n} \times[\vec{n} \times \vec{\beta}(t)]}{1-\vec{\beta}(t) \cdot \vec{n}}\right] e^{i \omega[t-\vec{n} \cdot \vec{r}(t) / c]} d t\right|^{2},
\end{aligned}
$$

the emitted radiation can be resolved into the $\sigma$ polarization (electric field in the plane of the electron motion and the observation direction) and $\pi$ polarization (electric field in the direction normal to the plane of the electron motion and the observation direction):

$$
\begin{aligned}
& \frac{d^{2} E_{\sigma}}{d \omega d \Omega}=\frac{e^{2}}{8 \pi^{2} c}\left|\int_{-\infty}^{\infty} \frac{d}{d t}\left[\frac{\vec{\varepsilon}_{\sigma} \cdot \vec{\beta}(t)}{1-\vec{\beta}(t) \cdot \vec{n}}\right] e^{i \omega(t-\vec{n} \cdot \vec{r}(t) / c)} d t\right|^{2}, \\
& \frac{d^{2} E_{\pi}}{d \omega d \Omega}=\frac{e^{2}}{8 \pi^{2} c}\left|\int_{-\infty}^{\infty} \frac{d}{d t}\left[\frac{\vec{\varepsilon}_{\pi} \cdot \vec{\beta}(t)}{1-\vec{\beta}(t) \cdot \vec{n}}\right] e^{i \omega(t-\vec{n} \cdot \vec{r}(t) / c)} d t\right|^{2}
\end{aligned}
$$

where $\quad \vec{\varepsilon}_{\sigma}=(-\sin \phi, \cos \phi, 0), \quad \vec{\varepsilon}_{\pi}=(\cos \theta \cos \phi$, $\cos \theta \sin \phi,-\sin \theta$ ), and the unit vector in the observation direction in the usual coordinates is $\vec{n}=$ $(\sin \theta \cos \phi, \sin \theta \sin \phi, \cos \theta)$.

In general 


$$
\begin{aligned}
& \frac{d^{2} E_{\sigma}}{d \omega d \Omega}=\frac{e^{2}}{8 \pi^{2} c}\left|\int_{-\infty}^{\infty} \frac{d}{d t}\left[\frac{-\beta_{x}(t) \sin \phi+\beta_{y}(t) \cos \phi}{1-\beta_{x}(t) \sin \theta \cos \phi-\beta_{y}(t) \sin \theta \sin \phi-\beta_{z}(t) \cos \theta}\right] e^{i \omega(t-\vec{n} \cdot \vec{r}(t) / c)} d t\right|^{2}, \\
& \frac{d^{2} E_{\pi}}{d \omega d \Omega}=\frac{e^{2}}{8 \pi^{2} c}\left|\int_{-\infty}^{\infty} \frac{d}{d t}\left[\frac{\beta_{x}(t) \cos \theta \cos \phi+\beta_{y}(t) \cos \theta \sin \phi-\beta_{z}(t) \sin \theta}{1-\beta_{x}(t) \sin \theta \cos \phi-\beta_{y}(t) \sin \theta \sin \phi-\beta_{z}(t) \cos \theta}\right] e^{i \omega(t-\vec{n} \cdot \vec{r}(t) / c)} d t\right|^{2} .
\end{aligned}
$$

Often, for example in [7], these expressions are integrated by parts in order to simplify, but such a procedure is not rigorously justified for the term proportional to the longitudinal velocity because the longitudinal velocity does not vanish when $t \rightarrow \pm \infty$. It is necessary to retain this more general form in order to rigorously evaluate the integrals with the field models which shall be calculated subsequently. Because the acceleration will be different from zero in a finite region, it is necessary to integrate numerically only over a finite domain. The far-field assumption is implicit in the assumption that $\vec{n}$ is constant and thus the observation direction is sensibly constant in the region where the acceleration is taking place. For the specific case of a linearly polarized magnetic device that deflects only in the $x$ direction, after the integrals are converted to integrals over the independent variable $z$, the distributions simplify to

$$
\begin{aligned}
& \frac{d^{2} E_{\sigma}}{d \omega d \Omega}=\frac{e^{2}}{8 \pi^{2} c}\left|\int_{-\infty}^{\infty} \frac{d}{d z}\left[\frac{-\beta_{x}(z) \sin \phi}{1-\beta_{x}(z) \sin \theta \cos \phi-\beta_{z}(z) \cos \theta}\right] e^{i \omega(t(z)-\vec{n} \cdot \vec{r}(z) / c)} d z\right|^{2}, \\
& \frac{d^{2} E_{\pi}}{d \omega d \Omega}=\frac{e^{2}}{8 \pi^{2} c}\left|\int_{-\infty}^{\infty} \frac{d}{d z}\left[\frac{\beta_{x}(z) \cos \theta \cos \phi-\beta_{z}(z) \sin \theta}{1-\beta_{x}(z) \sin \theta \cos \phi-\beta_{z}(z) \cos \theta}\right] e^{i \omega(t(z)-\vec{n} \cdot \vec{r}(z) / c)} d z\right|^{2} .
\end{aligned}
$$

These expressions may be manipulated into final form as follows. Assuming that $\beta_{x}(z)$ vanishes before and after the interaction with the magnetic device (the device does not deflect the electron), integration by parts, as in the usual calculation, is rigorously justified and

$$
\frac{d^{2} E_{\sigma}}{d \omega d \Omega}=\frac{e^{2} \omega^{2}}{8 \pi^{2} c^{3}}\left|\int_{-\infty}^{\infty} \frac{\beta_{x}(z) \sin \phi}{\beta_{z}(z)} e^{i \varphi(z)} d z\right|^{2}
$$

where the proper retarded phase is

$$
\begin{aligned}
\varphi(z)= & \frac{\omega\left(1-\beta_{z 0} \cos \theta\right) z}{\beta_{z 0} c}+\frac{\omega}{c} \int_{-\infty}^{z}\left[\frac{1}{\beta_{z}\left(z^{\prime}\right)}-\frac{1}{\beta_{z 0}}\right] d z^{\prime} \\
& -\frac{\omega \sin \theta \cos \phi}{c} \int_{-\infty}^{z} \frac{a_{x}\left(z^{\prime}\right) / \gamma_{0}}{\sqrt{\beta_{z 0}^{2}-a_{x}^{2}\left(z^{\prime}\right) / \gamma_{0}^{2}}} d z^{\prime}
\end{aligned}
$$

For the $\pi$ polarization the first term may also be rigorously integrated by parts, but the second term cannot be integrated so simply because the longitudinal velocity does not vanish as $z \rightarrow \pm \infty$. Integrating by parts the first term and directly differentiating the second term in the expression for the spectrum yields

$$
\begin{aligned}
\frac{d^{2} E_{\pi}}{d \omega d \Omega}= & \frac{e^{2} \omega^{2}}{8 \pi^{2} c^{3}} \mid \int_{-\infty}^{\infty}\left[\frac{-i \beta_{x}(z) \cos \theta \cos \phi}{\beta_{z}(z)}\right. \\
& \left.-\frac{\ddot{\varphi} \sin \theta}{(d \varphi / d z)^{2}}\right]\left.e^{i \varphi(z)} d z\right|^{2} .
\end{aligned}
$$

Writing

$$
\frac{d \varphi}{d z}=\frac{\omega}{c}\left\{\frac{\left(1-\beta_{z 0} \cos \theta\right)}{\beta_{z 0}}+f(z)\right\}
$$

where

$$
f(z)=\frac{1}{\beta_{z}(z)}-\frac{1}{\beta_{z 0}}-\sin \theta \cos \phi \frac{a_{x}(z) / \gamma_{0}}{\sqrt{\beta_{z 0}^{2}-a_{x}^{2}(z) / \gamma_{0}^{2}}}
$$

does vanish as $z \rightarrow \pm \infty$, we may now rigorously integrate by parts with vanishing boundary terms to obtain the identity

$$
\begin{aligned}
\int f(z) e^{i \varphi(z)} d z & =\int \frac{f}{i d \varphi / d z} \frac{d}{d z} e^{i \varphi} d z \\
& =\int\left[\frac{f \ddot{\varphi}}{i(d \varphi / d z)^{2}}-\frac{\dot{f}}{i d \varphi / d z}\right] e^{i \varphi} d z \\
& =-\frac{1-\beta_{0 z} \cos \theta}{\beta_{0 z}} \int \frac{\ddot{\varphi}}{i(d \varphi / d z)^{2}} e^{i \varphi} d z .
\end{aligned}
$$

So

$$
\begin{aligned}
\frac{d^{2} E_{\pi}}{d \omega d \Omega}= & \frac{e^{2} \omega^{2}}{8 \pi^{2} c^{3}} \mid \int_{-\infty}^{\infty}\left[\frac{\beta_{x}(z) \cos \theta \cos \phi}{\beta_{z}(z)}\right. \\
& \left.+\frac{f(z) \beta_{0 z} \sin \theta}{1-\beta_{0 z} \cos \theta}\right]\left.e^{i \varphi(z)} d z\right|^{2}
\end{aligned}
$$

and the radiation spectra can be summarized in the final forms

$$
\begin{aligned}
\frac{d^{2} E_{\sigma}}{d \omega d \Omega}= & \frac{e^{2} \omega^{2}}{8 \pi^{2} c^{3}}\left|D_{t}(\omega ; \theta, \phi)\right|^{2} \sin ^{2} \phi, \\
\frac{d^{2} E_{\pi}}{d \omega d \Omega}= & \frac{e^{2} \omega^{2}}{8 \pi^{2} c^{3}} \mid D_{t}(\omega ; \theta, \phi) \frac{\cos \theta-\beta_{z 0}}{1-\beta_{z 0} \cos \theta} \cos \phi \\
& +\left.D_{l}(\omega ; \theta, \phi) \frac{\beta_{z 0} \sin \theta}{1-\beta_{z 0} \cos \theta}\right|^{2},
\end{aligned}
$$

where 


$$
\begin{aligned}
& D_{t}(\omega ; \theta, \phi)=\int_{-\infty}^{\infty} \frac{a_{x}(z) / \gamma_{0}}{\sqrt{\beta_{z 0}^{2}-a_{x}^{2}(z) / \gamma_{0}^{2}}} \operatorname{expi\varphi (z)dz,} \\
& D_{l}(\omega ; \theta, \phi)=\int_{-\infty}^{\infty}\left[\frac{1}{\beta_{z}(z)}-\frac{1}{\beta_{z 0}}\right] \exp i \varphi(z) d z .
\end{aligned}
$$

In the low-field limit $a_{x} \ll 1$, and $D_{l} \ll D_{t}$ because $D_{l}$ involves an addition power of $a_{x}$ compared to $D_{t}$. Then the integral for $D_{t}$ reduces to the standard Fourier integral over the vector potential function, but at a Doppler up-shifted frequency. The spectral angular distributions reduce to the low-field distribution referred to in the introduction that has been known for many decades [1]. In the case that $a_{x}$ is purely sinusoidal, these expressions reduce to the usual undulator design equations including the frequency shifts and harmonic generation that arise at high-field strength parameters. Such frequency shifts are found in the second term of the phase function by expanding the longitudinal velocity difference to leading order and can be shown to become proportional to $1+K^{2} / 2$ in the forward direction, where $K=\left|a_{x}\right|$. This redshifting is well known in insertion device and free-electron laser (FEL) theory.

When no assumptions are made about the smallness of the field strength, and the integrals are completed numerically, one has an exact theory, in the far-field limit, which can cover the case of interest stated in the introduction. Given a model of the electromagnetic deflection of the electron as embodied in the vector potential function $a_{x}$, one can now compute the radiation spectrum in cases where the magnetic device is both short and the magnetic field strength parameter is comparable to or greater than 1 . The main additional physics captured in this formulation is to properly track the slowing down of the longitudinal velocity of the electrons as they move through the magnetic field of the magnetic device, which will tend to redshift and broaden the radiation spectrum.

\section{LARMOR'S THEOREM FOR THE TOTAL ENERGY RADIATED}

In this section an expression for the total radiated energy is given. The relativistic generalization of Larmor's theorem is given by Barut [8] as

$$
\frac{d E}{d \tau}=\frac{2}{3} \frac{e^{2}}{c^{3}} \frac{d t}{d \tau} \frac{d}{d \tau}\left(\frac{d x^{\mu}}{d \tau}\right) \frac{d}{d \tau}\left(\frac{d x_{\mu}}{d \tau}\right)
$$

where $\tau$ is the proper time. Because $d t=\gamma d \tau=\gamma_{0} d \tau$, and $z$ can be made the independent variable,

$$
\frac{d E}{d z}=\frac{2}{3} e^{2} \gamma_{0}^{4} \beta_{z}(z) \frac{d \beta^{\mu}}{d z} \frac{d \beta_{\mu}}{d z}
$$

Using Eqs. (2) and (3) above,

$$
\begin{aligned}
\frac{d \beta_{x}}{d z} & =\frac{1}{\gamma_{0}} \frac{d a_{x}}{d z}, \quad \frac{d \beta_{y}}{d z}=\frac{1}{\gamma_{0}} \frac{d a_{y}}{d z}, \\
\frac{d \beta_{z}}{d z} & =-\frac{1}{\gamma_{0}^{2}} \frac{a_{x} \frac{d a_{x}}{d z}+a_{y} \frac{d a_{y}}{d z}}{\sqrt{\beta_{z 0}^{2}-a_{x}^{2} / \gamma_{0}^{2}-a_{y}^{2} / \gamma_{0}^{2}}},
\end{aligned}
$$

and

$$
\frac{d E}{d z}=\frac{2}{3} e^{2}\left[\gamma_{0}^{2} \beta_{z}\left(\frac{d a_{x}}{d z}\right)^{2}+\gamma_{0}^{2} \beta_{z}\left(\frac{d a_{y}}{d z}\right)^{2}+\frac{a^{2} a^{12}}{\beta_{z}}\right],
$$

where $a=\sqrt{a_{x}^{2}+a_{y}^{2}}$. There is consequently a general total energy sum rule

$$
\begin{aligned}
E= & \frac{2}{3} e^{2} \int_{-\infty}^{\infty} \sqrt{\beta_{z 0}^{2}-a^{2} / \gamma_{0}^{2}}\left[\gamma_{0}^{2}\left(\frac{d a_{x}}{d z}\right)^{2}+\gamma_{0}^{2}\left(\frac{d a_{y}}{d z}\right)^{2}\right. \\
& \left.+\frac{a^{2} a^{\prime 2}}{\beta_{z 0}^{2}-a^{2} / \gamma_{0}^{2}}\right] d z
\end{aligned}
$$

which reduces to

$$
\begin{aligned}
E= & \frac{2}{3} e^{2} \int_{-\infty}^{\infty} \sqrt{\beta_{z 0}^{2}-a_{x}^{2} / \gamma_{0}^{2}}\left[\gamma_{0}^{2}\left(\frac{d a_{x}}{d z}\right)^{2}\right. \\
& \left.+\frac{a_{x}^{2}\left(d a_{x} / d z\right)^{2}}{\beta_{z 0}^{2}-a_{x}^{2} / \gamma_{0}^{2}}\right] d z
\end{aligned}
$$

in the case of a linearly polarized magnet which has a single vector potential component $a_{x}$. This integral is easily calculated numerically and can be estimated analytically in many interesting cases.

\section{APPLICATIONS TO SHORT HIGH-FIELD MAGNETS}

In the rest of this paper, only the simplest cases of magnetic devices on a straight line downstream of a linac will be considered. The results may not have direct application to cases, as in an insertion device in a storage ring, where there are bends both before and after the insertion device. The generation of spontaneous radiation by the edges of the bends, although certainly calculable using the ideas presented in this paper, are not covered in the relatively simple models that will be analyzed subsequently. Likewise, the calculation of spontaneous emission from a magnetic device downstream of a bending magnet should properly include the contributions from the edge field of the bending magnet.

The simplest magnetic device one can think of is one that has a single bump corresponding to one-half a period of oscillation. Unfortunately, a single region with high magnetic field of one sign will deflect the electron beam. In order for the beam to be undeflected and unmoved by the magnetic device it is sufficient that the electron orbit vanishes as $z \rightarrow \infty$. A convenient approximate functional form for the orbit which accomplishes this boundary con- 
dition is

$$
x(z)=-x_{0} \exp \left(-z^{2} / 2 \sigma^{2}\right),
$$

where $x_{0}$ quantifies the size of the transverse motion and $\sigma$ is the magnetic field scale length. For high energy electrons where $\beta_{z} \approx 1$, the vector potential to produce such an orbit is

$$
e A_{x}(z) / \gamma_{0} m c^{2} \approx \frac{d x}{d z}=\frac{x_{0} z}{\sigma^{2}} \exp \left(-z^{2} / 2 \sigma^{2}\right)
$$

with magnetic field

$$
B_{y}(z)=\frac{\partial A_{x}}{\partial z}=\frac{\gamma_{0} m c^{2}}{e} \frac{x_{0}}{\sigma^{2}}\left(1-\frac{z^{2}}{\sigma^{2}}\right) \exp \left(-z^{2} / 2 \sigma^{2}\right) .
$$

The maximum magnetic field, $B_{\max }$ is $\gamma_{0} m c^{2} x_{0} / e \sigma^{2}$, and the orbit becomes

$$
x(z)=-\left(K \sigma / \gamma_{0}\right) \exp \left(-z^{2} / 2 \sigma^{2}\right)
$$

where the field strength parameter, $K$, is defined to be

$$
K=\frac{e B_{\max } \sigma}{m c^{2}} .
$$

Figure 1 shows the magnitude of the effective motion spectrum in the forward direction normalized to the magnet length scale parameter, $D_{t}(\omega ; \theta=0) / \sigma$, for three values of the field strength when $\gamma_{0}=100$. The frequency is scaled to the frequency at which the curve is maximum at low values of the field strength parameter, $\omega_{0} \approx 2 \gamma_{0}^{2} c / \sigma$. At low values of the field strength parameter the motion spectrum follows the functional form

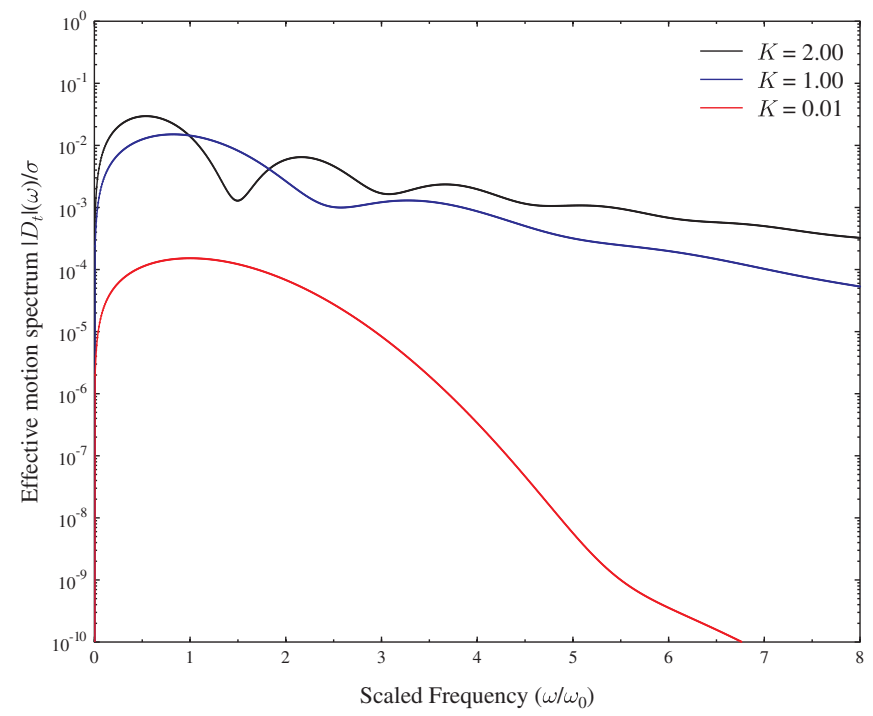

FIG. 1. (Color) Effective motion spectrum for wide-band source.

$$
\begin{aligned}
\frac{D_{t}}{\sigma} & =i \frac{\sqrt{2 \pi} K}{\gamma_{0}}\left(\frac{\omega}{\omega_{0}}\right) \exp \left(-\frac{\omega^{2}}{2 \omega_{0}^{2}}\right) \\
\omega_{0} & =\frac{c \beta_{z 0}}{\left(1-\beta_{z 0} \cos \theta\right) \sigma}
\end{aligned}
$$

which follows easily by taking the proper Fourier integral. Figure 2 shows the full spectrum emitted at zero angle to the magnet axis. At low-field strengths the $\frac{1}{2}$ power points for the spectrum are at the points 0.62 and 1.44 times the frequency of maximum emission. As the field strength increases the spectrum redshifts, as is usual in conventional undulator theory, from the slowing down of the electron in the longitudinal direction as it enters the magnetic field. This effect causes the spectrum maximum to shift to $0.74 \omega_{0}$ when $K=2$, with a lower $\frac{1}{2}$ maximum point $0.46 \omega_{0}$ and an upper $\frac{1}{2}$ maximum point at $1.01 \omega_{0}$. At higher values of the field strength the spectra also broaden appreciably; the harmonic emission, which also has a very wide bandwidth, increases substantially at larger values of the field strength.

At low $K$, the maximum in the energy spectrum is at the frequency $\omega_{\max }=2 \pi f_{\max }=\sqrt{2} \omega_{0}$ which in the forward direction has a wavelength of

$$
\lambda_{\max }=\frac{\left(1-\beta_{z 0}\right) 2 \pi \sigma}{\sqrt{2} \beta_{z 0}} \approx \frac{2 \pi \sigma}{2^{3 / 2} \gamma_{0}^{2}},
$$

in terms of the scale length for the magnetic field. The energy emitted per electron may be estimated using the total energy sum rule

$$
\begin{aligned}
E_{\mathrm{emitted}} & \approx \frac{2}{3} e^{2} \gamma^{2} \int_{-\infty}^{\infty}\left(\frac{d a_{x}}{d z}\right)^{2} d z=\frac{\sqrt{\pi}}{2} e^{2} \gamma_{0}^{2} K^{2} / \sigma \\
& =\frac{\sqrt{\pi}}{2} \gamma_{0} K^{2} \frac{r_{e}}{\sigma} E,
\end{aligned}
$$

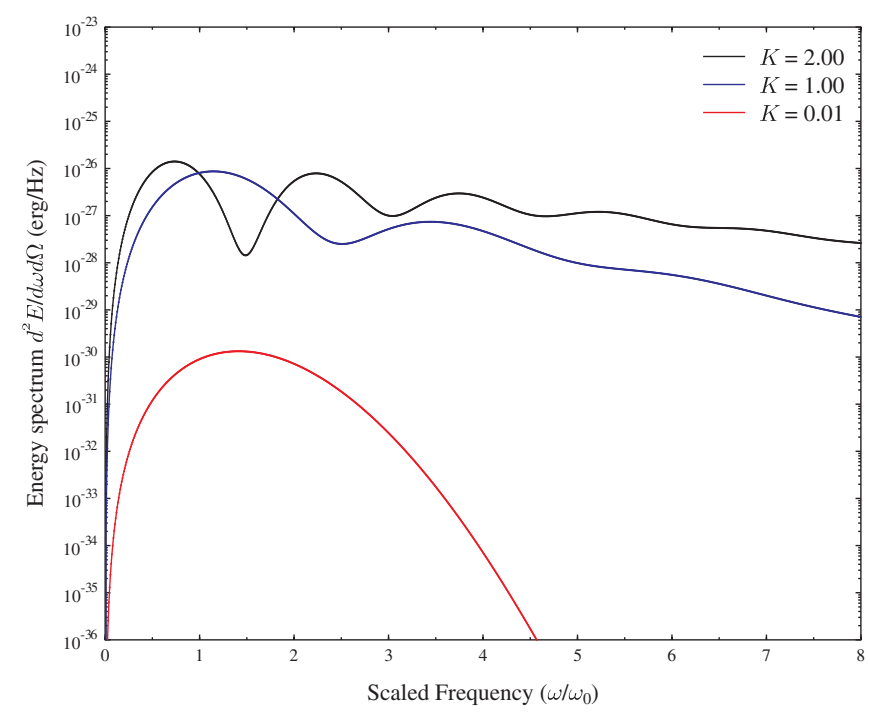

FIG. 2. (Color) Forward energy spectrum for wide-band source. 
where $E_{\text {emitted }}$ is the incoherent energy emitted by a single electron of total energy $E=\gamma_{0} m c^{2}$, and $r_{e}$ is the classical electron radius. A magnet of such a design is interesting as a wide bandwidth source of coherent $\mathrm{THz}$ electromagnetic radiation [9].

A Gaussian amplitude modulated sinusoidal motion gives another example that is fairly easy to calculate, and gives an analytically tractable low-field strength limit. If

$$
x(z)=-x_{0} \cos (2 \pi z / \lambda) \exp \left(-z^{2} / 2 \sigma^{2}\right),
$$

the vector potential to produce such an orbit is

$$
\begin{aligned}
e A_{x}(z) / \gamma_{0} m c^{2} \approx & \frac{d x}{d z} \\
= & \frac{x_{0}}{\sigma}\left[\frac{2 \pi \sigma}{\lambda} \sin \frac{2 \pi z}{\lambda}+\frac{z}{\sigma} \cos \frac{2 \pi z}{\lambda}\right] \\
& \times \exp \left(-z^{2} / 2 \sigma^{2}\right),
\end{aligned}
$$

with magnetic field

$$
\begin{aligned}
B_{y}(z)= & \frac{\partial A_{x}}{\partial z} \\
= & \frac{\gamma_{0} m c^{2}}{e} \frac{x_{0}}{\sigma^{2}}\left\{\left[1+\left(\frac{2 \pi \sigma}{\lambda}\right)^{2}-\frac{z^{2}}{\sigma^{2}}\right] \cos \frac{2 \pi z}{\lambda}\right. \\
& \left.-2 \frac{2 \pi z}{\lambda} \sin \frac{2 \pi z}{\lambda}\right\} \exp \left(-z^{2} / 2 \sigma^{2}\right) .
\end{aligned}
$$

The maximum magnetic field, $B_{\max }$ is $\gamma_{0} m c^{2} x_{0}(1+$ $\left.(2 \pi \sigma / \lambda)^{2}\right) / e \sigma^{2}$, and the orbit becomes

$$
x(z)=-\left(K \sigma_{\text {eff }} / \gamma_{0}\right) \cos (2 \pi z / \lambda) \exp \left(-z^{2} / 2 \sigma^{2}\right),
$$

where the field strength parameter $K$ is now

$$
K=\frac{e B_{\max } \sigma_{\mathrm{eff}}}{m c^{2}},
$$

and the effective scale length is

$$
\sigma_{\text {eff }}=\frac{\sigma \lambda}{\sqrt{\lambda^{2}+(2 \pi)^{2} \sigma^{2}}} .
$$

The energy emitted per electron may be estimated using the total energy sum rule, Parseval's theorem from Fourier transform theory, and the expression for the Fourier transform of the vector potential function which is easily obtained by completing the square in the Gaussian integral:

$$
\begin{aligned}
E_{\text {emitted }} \approx & \frac{2}{3} e^{2} \gamma^{2} \int_{-\infty}^{\infty}\left(\frac{d a_{x}}{d z}\right)^{2} d z \\
= & \frac{\sqrt{\pi}}{3} \gamma_{0} K^{2} \frac{r_{e}}{\sigma} E \frac{\lambda^{2}}{\lambda^{2}+4 \pi^{2} \sigma^{2}} \times\left[\frac{3}{4}(1\right. \\
& \left.\left.+\exp \left[-\left(\frac{2 \pi \sigma}{\lambda}\right)^{2}\right]\right)+3\left(\frac{2 \pi \sigma}{\lambda}\right)^{2}+\left(\frac{2 \pi \sigma}{\lambda}\right)^{4}\right]
\end{aligned}
$$

Note that this result reduces to that in Eq. (5) in the limit $\lambda \rightarrow \infty$. More importantly, it reveals the proper scaling when $\sigma \gg \lambda$. For fixed $\lambda$, because the number of wiggles in the field is proportional to $\sigma$, the total energy radiated goes as the number of wiggles.

Figure 3 shows the magnitude of the effective motion spectrum in the forward direction normalized to the magnet length scale parameter, $D_{t}(\omega ; \theta=0) / \lambda$, for two values of the field strength in the magnet when $\sigma=8.156 \lambda$. Again, the frequency is scaled to the frequency at which the curve is maximum at low values of the field strength parameter, but in this case the emission frequency is Doppler up-shifted to $\omega_{0} \approx 2 \gamma_{0}^{2}(2 \pi c / \lambda)$. At low values of the field strength parameter the motion spectrum follows the functional form

$$
\begin{aligned}
\frac{D_{t}}{\lambda}= & i \frac{(2 \pi)^{3 / 2}}{2 \gamma_{0}} \frac{K}{\sqrt{1+4 \pi^{2} \sigma^{2} / \lambda^{2}}}\left(\frac{\omega}{\omega_{0}}\right)\left(\frac{\sigma}{\lambda}\right)^{2} \\
& \times\left[\exp \left(-\frac{\left(\omega+\omega_{0}\right)^{2}}{2 \sigma_{\omega}^{2}}\right)+\exp \left(-\frac{\left(\omega-\omega_{0}\right)^{2}}{2 \sigma_{\omega}^{2}}\right)\right], \\
\omega_{0}= & \frac{2 \pi c \beta_{z 0}}{\left(1-\beta_{z 0} \cos \theta\right) \lambda} \quad \sigma_{\omega}=\frac{c \beta_{z 0}}{\left(1-\beta_{z 0} \cos \theta\right) \sigma},
\end{aligned}
$$

which follows easily by taking the proper Fourier integral. Figure 4 shows the full emitted spectrum at zero angle to the magnet axis. As the field strength increases the spectrum redshifts, as is usual in conventional undulator theory, from the slowing down of the electron in the longitudinal direction as it enters the magnetic field. It also broadens appreciably. The harmonic emission, which also has a very wide bandwidth, increases substantially at larger values of the field strength.

In conclusion, in this paper a convenient theoretical description has been used to obtain the spontaneous emission from a couple of short, high-field magnetic devices. These results are similar to those obtained in high intensity pulsed laser Thomson scattering, where ponderomotive

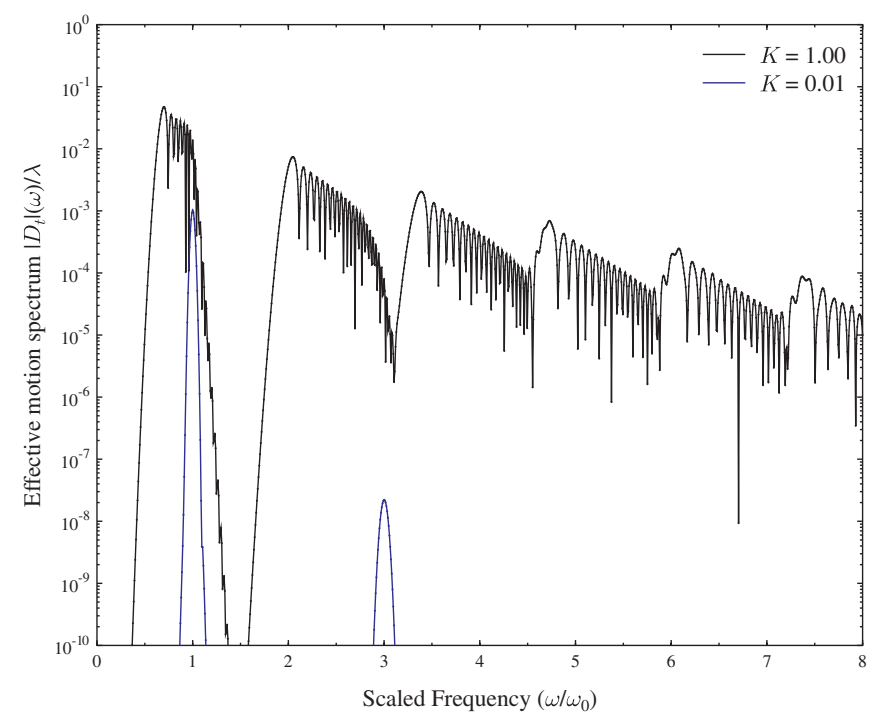

FIG. 3. (Color) Effective motion spectrum for Gaussian amplitude modulated magnet. 


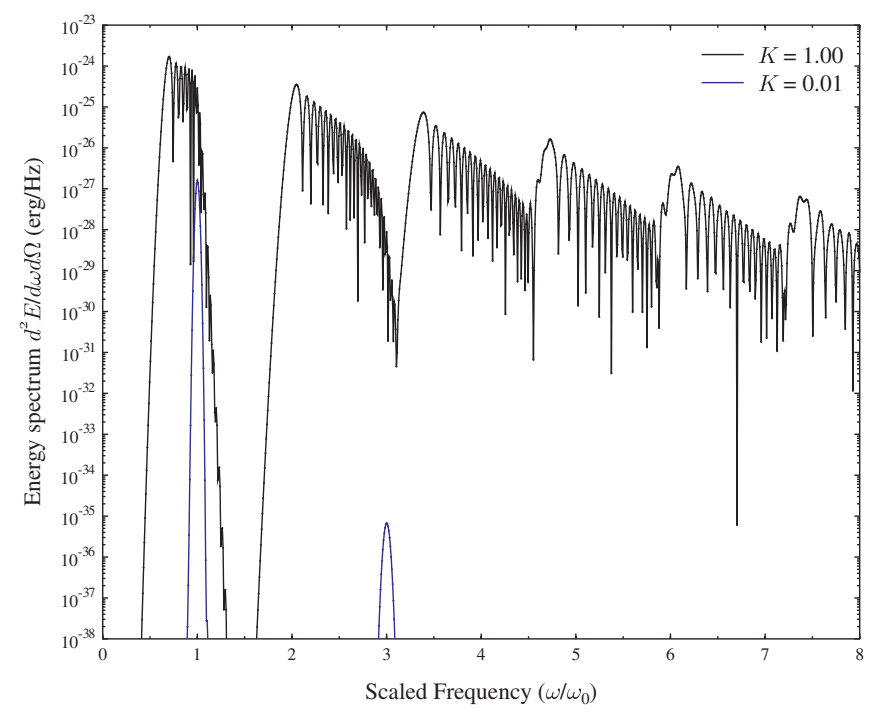

FIG. 4. (Color) Forward energy spectrum for Gaussian amplitude modulated magnet.

broadening has been found. The formalism allows the problem of harmonic generation in short magnets to be calculated exactly, in the far-field limit. The spontaneous radiation spectrum emitted from two specific simple designs is investigated in detail: a short, wide-band device that may be useful as a $\mathrm{THz}$ radiation source and a Gaussian amplitude modulated sinusoidal magnetic device. The latter type of device certainly exhibits broadening in some parameter regimes. Similar physical effects may be relevant in the analysis of FEL radiation from tapered wigglers.

\section{ACKNOWLEDGMENTS}

Valuable discussions with Adnan Doyuran on the numerical solution of equations of the type found in this paper are gratefully acknowledged. This work supported by the United States Department of Energy under Contract No. DE-AC05-84ER40150.

[1] R. Coisson, Phys. Rev. A 20, 524 (1979).

[2] R. Bossart, J. Bosser, L.Burnod, R. Coisson, E. D’Amico, A. Hofmann, and J. Mann, Nucl. Instrum. Methods 164, 375 (1979).

[3] E. S. Sarachik and G. T. Schappert, Phys. Rev. D 1, 2738 (1970).

[4] Sally K. Ride, Eric Esarey, and Michael Baine, Phys. Rev. E 52, 5425 (1995).

[5] G. A. Krafft, Phys. Rev. Lett. 92, 204802 (2004).

[6] G. A. Krafft, A. Doyuran, and J. Rosenzwieg, Phys. Rev. E 72, 056502 (2005).

[7] J.D. Jackson, Classical Electrodynamics (Wiley, New York, 1975), 2nd ed., Chap. 14, p. 670.

[8] A.O. Barut, Electrodynamics and Classical Theory of Fields and Particles (MacMillan, New York, 1964), Eq. 5.52.

[9] G. A. Krafft, Phys. Rev. ST Accel. Beams 7, 060704 (2004). 